ACT/ACT. Of these, 14 had abnormal LCI but only 4 had abnormal $\mathrm{FEV}_{1}$. 8/28 asthmatics had raised LCI despite normal $\mathrm{FEV}_{1}$.

Conclusions LCI, a measure of ventilation heterogeneity, is raised in a high proportion of children with severe asthma. Most children with raised LCI had normal spirometry. This suggests that LCI is more sensitive to detect lung function deficits in asthma compared to spirometry. LCI also correlates well with symptom control. MBNW and LCI may be useful in the monitoring of children with severe asthma.

\section{P90 HOW DOES BMI STATUS INFLUENCE SPIROMETRY AND RESPIRATORY MUSCLE STRENGTH IN CHILDREN?}

${ }^{1} \mathrm{GSJ}$ Duncan, ${ }^{1} \mathrm{NT}$ Gharbawi, ${ }^{2} \mathrm{M}$ Viskaduraki, ${ }^{1} \mathrm{EA}$ Gaillard, ${ }^{1} \mathrm{CS}$ Beardsmore. ${ }^{1}$ Department of Infection, Immunity and Inflammation (Child Health), University of Leicester, Leicester, UK; ${ }^{2}$ Bioinformatics and Biostatistics Hub, College of Medicine, Biology and Psychology, University of Leicester, Leicester, UK

\subsection{6/thoraxjnl-2017-210983.232}

Introduction While BMI correlates positively with spirometry during childhood, young children who are overweight or obese have been shown to have a reduced $\mathrm{FEV}_{1} / \mathrm{FVC}$ compared to their peers. ${ }^{1}$ In childhood, obesity has been shown to have a negative effect upon inspiratory muscle strength. ${ }^{2}$

Aims To assess whether there are differences in spirometry of children of varying BMI status and whether this relates to respiratory muscle strength.

Methods Within schools, we measured each child's height, weight, and spirometry. Respiratory muscle strength was assessed via maximal inspiratory and expiratory pressures (MIP/MEP). The child breathed through a pneumotachograph attached to a shutter. To measure MIP, the child exhaled maximally and the shutter was activated. The child made an inspiratory effort against the shutter and peak pressure was recorded. The test was repeated several times. Measurements of MEP were similar, except that the child inhaled maximally and then made a forceful expiratory effort. We calculated BMI and grouped children by centile score into underweight, healthy, overweight or obese, using epidemiological cut-offs. Results were adjusted for age and height via an ANCOVA.

Results We studied 297 children (5-11 year). We obtained data for spirometry in 258, MIP in 231 and MEP in 262. Mean adjusted values are shown (Table). All individual parameters showed significant positive correlation with BMI, while $\mathrm{FEV}_{1} / \mathrm{FVC}$ was significantly negatively correlated with BMI. The obese group had a significantly greater adjusted mean value for MEP than the healthy group and a significantly greater mean adjusted value for FVC than the underweight group, while having a significantly lower mean adjusted $\mathrm{FEV}_{1} /$ FVC than both healthy and underweight groups.

Conclusion Despite having the greatest adjusted mean value for expiratory muscle strength and vital capacity, the obese group demonstrated the lowest adjusted mean $\mathrm{FEV}_{1} / \mathrm{FVC}$, indicating a potential alteration in respiratory flow dynamics for children of greater BMI.

\section{REFERENCES}

1. Tantisira, et al. Thorax 2003, Dec;58(12):1036-41.

2. da Rosa, et al. Rev Paul Pediatr 2014, Jun;32(2):250-5.
Abstract P90 Table 1

\begin{tabular}{lllll}
\hline & Underweight & Healthy & Overweight & Obese \\
\hline FEV $_{1}(\mathrm{~L})$ & 1.55 & 1.69 & 1.69 & 1.67 \\
FVC (L) & 1.70 & 1.90 & 1.94 & 1.97 \\
FEV $_{1} / \mathrm{FVC}$ & 91.2 & 89.1 & 87.7 & 85.7 \\
$(\%)$ & & & \\
MIP (kPa) & 6.89 & 7.07 & 7.68 & 7.57 \\
MEP (kPa) & 6.01 & 6.29 & 6.54 & 7.12 \\
\hline
\end{tabular}

\section{Multi-morbidity in COPD}

\section{P91 HIGH PREVALENCE OF VITAMIN D DEFICIENCY AMONGST PATIENTS WITH COPD IN THE NORTH EAST. HIGHLIGHTING A DEFICIENCY AND NEED FOR IMPROVED ASSESSMENT}

H Tedd, K Conroy, A Mitchell, Y Shanshal, H Curtis. Queen Elizabeth Hospital, Gateshead, UK

\subsection{6/thoraxjnl-2017-210983.233}

Background Vitamin D plays a key role in osteoporosis and also contributes to sarcopenia, muscle weakness, fatigue and depression. Patients with COPD are likely to be at higher risk of Vitamin D deficiency due to reduced mobility especially outdoors, with previous studies in the London area demonstrating prevalence rates around $60 \% .{ }^{1}$ However, within our population group in the North East of England, little is known about the prevalence of Vitamin D deficiency.

Aims To identify the prevalence of serum 25-hydroxyvitamin D (25(OH)-D) deficiency in patients admitted with an acute exacerbation of COPD.

Method We identified 50 patients admitted with an exacerbation of COPD. Data on demographics and prescription of vitamin D supplementation was recorded. 25(OH)-D titres were measured.

Results 50 patients included, mean age 73.6 years (age range 45-95 years). $44 \%$ of patients were prescribed vitamin D supplementation (95\% of supplementation was in the form of combined calcium and vitamin D). Overall $62 \%$ of patients were found to have low 25(OH)-D titres. Of those not taking vitamin D supplementation, only $14 \%$ of patient had sufficient $25(\mathrm{OH})$-D titres $(\geq 50 \mathrm{nmol} / \mathrm{L}) .11 \%$ were $25(\mathrm{OH})$-D insufficient $(30-50 \mathrm{nmol} / \mathrm{L}), \quad 57 \%$ were $25(\mathrm{OH})-\mathrm{D}$ deficient $(8-$ $30 \mathrm{nmol} / \mathrm{L})$ and $18 \%$ were profoundly deficient $(<8 \mathrm{nmol} / \mathrm{L})$. Of those patients taking vitamin D supplementation, $68 \%$ were found to have sufficient $25(\mathrm{OH})$-D titres, whilst $32 \%$ still had inadequate $25(\mathrm{OH})$-D highlighting potential issues with compliance or insufficient replacement.

Conclusions We have demonstrated a very high prevalence of vitamin D deficiency amongst our patients with COPD, with $86 \%$ of our patients having inadequate vitamin $\mathrm{D}$ titres who were not on vitamin D supplementation. This is leading them to increased exposure to the risks of vitamin D deficiency, including the impact on bone health in at already 'at-risk' population. In response to this, locally we are now measuring 25(OH)-D titres routinely on patients with COPD and prescribing vitamin D supplementation when indicated, forming 
part of our new multisystem, comprehensive, holistic assessment of COPD patients.

\section{REFERENCE}

1. Joliffe, et al. Prevalence, determinants and clinical correlates of Vitamin D deficiency in patients with chronic obstructive pulmonary disease in London, UK., 2017. J Steroid Biochem Mol Biochem.

\section{P92 EFFICACY OF BETA BLOCKERS PRESCRIBED AMONG COPD PATIENTS WITH CONCOMITANT HEART FAILURE}

${ }^{1} S$ Ashraf, ${ }^{2}$ A Ashraf. 'Pulmonology Department, Khyber Medical College/ Khyber Teaching Hospital; ${ }^{2}$ Cardiology Department, Khyber Medical College/Khyber Teaching Hospital

\subsection{6/thoraxjnl-2017-210983.234}

Background Due to common risk factors, there is considerable number of COPD patients who has concomitant heart failure. There is always reluctance in prescribing beta blockers in patients with COPD, though recent literature has supported the use of cardio-selective beta blockers among these patients. We conducted this study to determine the effect of cardioselective beta blockers on dyspnea grade and exacerbation rate among COPD patients with concomitant heart failure.

Methods This was a prospective cohort study among COPD patients with concomitant heart failure, conducted in a clinic during the last one year. Patients were recruited into 2 groups those who were prescribed cardio- selective beta blockers (group 1) and those managed without beta blockers (group 2). Patients were followed for one year. Outcomes measured were the reduction in MRC dyspnea grade and reduction in number of exacerbations in this year as compare to last year. Those patients having renal disease, liver disease, cancer, any Pneumonia leading to hospitalisation, stroke, etc. were excluded from the study.

Results Total of 95 patients (45 in group 1 and 50 in group 2 ), mean age $61.3 \pm 11$ years, BMI $27.5 \pm 6.8$, mean COPD exacerbation rate of $2.45 \pm 0.8$ were included in the study according to inclusion criteria. There was statistically significant difference in the two groups regarding their smoking history and BMI, though no difference in the gender distribution and mean COPD exacerbation rate in the last year. At the end of one year follow up, we found statistically significant difference in reduction in COPD exacerbation rate and reduction in dyspnea grade with $\mathrm{p}<0.05$.

Conclusion Cardio- selective beta blockers when prescribed among sub group of COPD patients who had concomitant heart failure may benefit in terms of reduction in dyspnea grade and reduction in COPD exacerbation rate.

\section{P93 'COPD: CT THORAX - FRIEND OR FOE': CLINICAL UTILITY OF CT THORAX IN DIAGNOSING COMORBIDITIES}

A Vohra, P Dalal, S Kaul. Harefield Hospital, Uxbridge, UK

\subsection{6/thoraxjnl-2017-210983.235}

Introduction Chronic obstructive pulmonary disease (COPD) is associated with several pulmonary and extra-pulmonary comorbidities. Comorbidities have a significant impact on health, healthcare services, and mortality in COPD patients, who have, on average, $\geq 4$ additional diseases. ${ }^{1}$ Earlier detection and treatment will lead to better patient outcomes. This study aims to demonstrate the added value of non-contrast CT Thorax in revealing previously unreported co-morbidities. Our hypothesis is CT Thorax is often requested in COPD patients primarily for co-existing lung disease however extra-pulmonary comorbidities are often under requested and under reported.

\section{Methods}

Setting Tertiary cardio thoracic centre

Study design Retrospective review 1000 non-contrast CT thorax scans in COPD patients. Using a pre-formed list of comorbidities (listed below), images were reviewed by a single operator. Pulmonary bronchiectasis, infection, lung cancer, ILD Extra-pulmonary Coronary artery calcification, Pulmonary artery diameter, hiatus hernia, vertebral fractures.

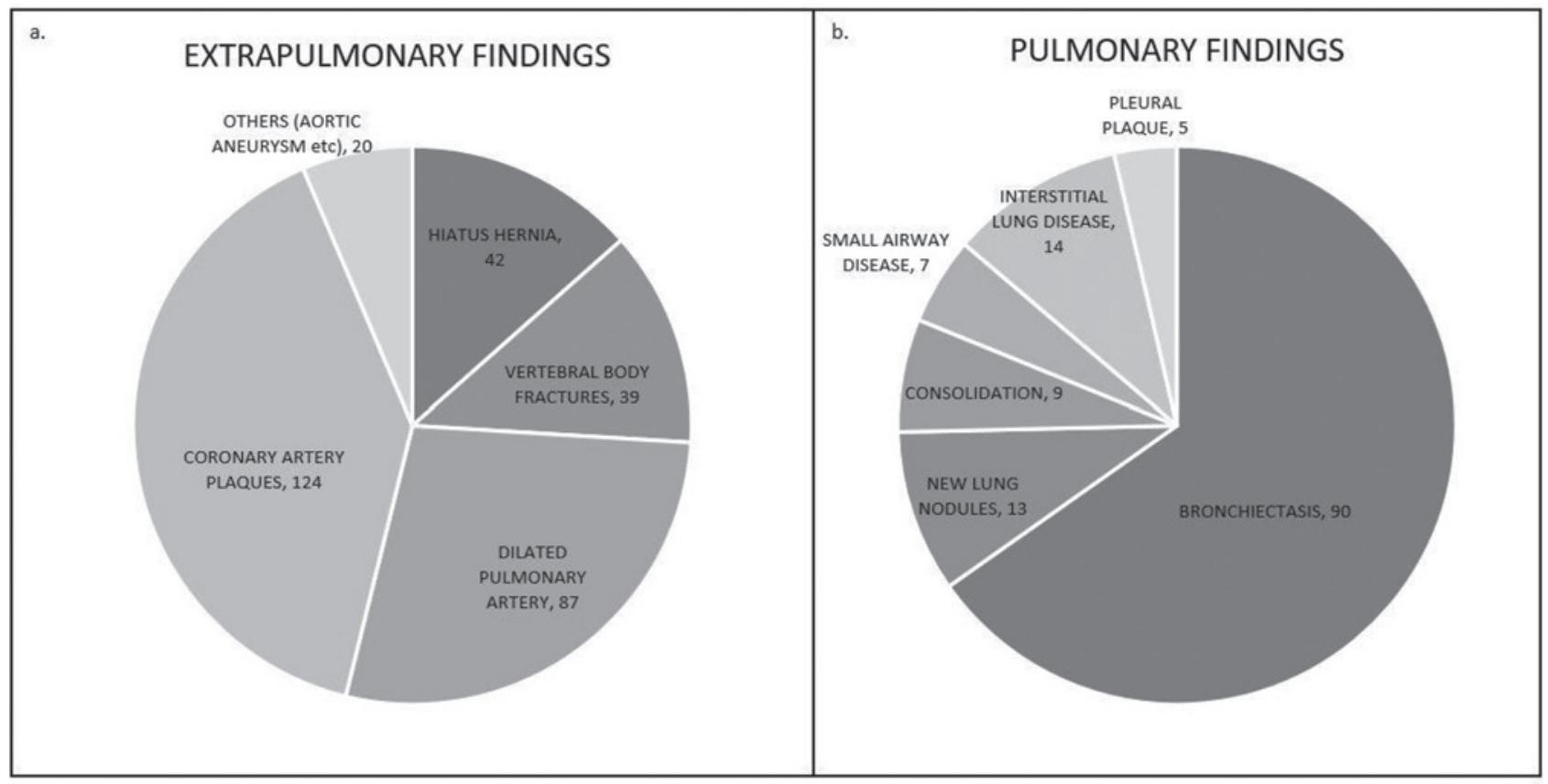

Abstract P93 Figure 1 Pie charts showing extra pulmonary (a) and pulmonary findings (b) on retrospective analysis of 227 CT scans. 